\title{
Differentiation of the Aging Process of PEDOT:PSS Films under Inert Helium and Ambient Atmosphere for Two Different Rates of Thermal Treatment
}

\author{
Sotirios Sakkopoulos, Evangelos Vitoratos \\ Department of Physics, University of Patras, Patras, Greece \\ Email: sakkop@physics,upatras.gr
}

Received September 25, 2013; revised October 25, 2013; accepted November 2, 2013

Copyright (C) 2014 Sotirios Sakkopoulos, Evangelos Vitoratos. This is an open access article distributed under the Creative Commons Attribution License, which permits unrestricted use, distribution, and reproduction in any medium, provided the original work is properly cited. In accordance of the Creative Commons Attribution License all Copyrights (C) 2014 are reserved for SCIRP and the owner of the intellectual property Sotirios Sakkopoulos, Evangelos Vitoratos. All Copyright (c) 2014 are guarded by law and by SCIRP as a guardian.

\section{ABSTRACT}

The conductivity difference $\Delta \sigma$ between two similar PEDOT:PSS films, $120 \mathrm{~nm}$ thick, heated by 5 and $15 \mathrm{~K} / \mathrm{min}$ from 80 to $440 \mathrm{~K}$, the one under inert $\mathrm{He}$ and the other under ambient air gives an approximate measure of the influence of oxygen and moisture on the conductivity vs temperature $T$. The resulting curves $\Delta \sigma=f(T)$ exhibit three different regions: at temperatures from 80 to $145 \mathrm{~K}$ for heating rate $5 \mathrm{~K} / \mathrm{min}$ and from 80 to $200 \mathrm{~K}$ for 15 $\mathrm{K} / \mathrm{min}$, an intense degradation of the sample under ambient atmosphere was revealed by the abrupt increase of the difference of the conductivities between He and atmospheric air. At intermediate temperatures from 145 to $380 \mathrm{~K}$ for $5 \mathrm{~K} / \mathrm{min}$ and 200 to $400 \mathrm{~K}$ for $15 \mathrm{~K} / \mathrm{min}$, the conductivity difference remains basically constant indicating a stabilization of the damage produced by oxygen and moisture. Finally, for temperatures higher than 380 to $400 \mathrm{~K}$ the degradation increases again. An explanation of this behavior is proposed based on the hydrophilic character of the PSS and the destructive role of oxygen at high temperatures. Moreover, the isotherms $\sigma=\sigma(\mathrm{t})$, where $t$ is the heating time, at $\mathbf{4 4 3} \mathrm{K}$, under inert $\mathrm{He}$ and under atmospheric air for one hour verify the significant role of oxygen and moisture on the electrical conductivity. Under He the conductivity increases monotonically with $t$, but under ambient air factors the conductivity competes with others decreasing it producing a maximum at about $t=10 \mathrm{~min}$.

\section{KEYWORDS}

Conducting Polymers; PEDOT:PSS; Thermal Treatment; Organic Electronics

\section{Introduction}

Poly(3,4-ethylenedioxythiophene) (PEDOT) is a $\pi$-conjugated polymer characterized by high conductivity and excellent optical transparency. PEDOT mixed with polystyrene sulphonate (PSS) forms a processible colloidal solution in water consisting of a random coil entanglement of hydrophilic insulating PSS chains with attached hydrophobic conductive PEDOT oligomers, forming conductive grains 30 - $50 \mathrm{~nm}$ in diameter. These grains consist of a PEDOT-rich core surrounded by a PSS-rich shell [1]. Adhesion between the PEDOT:PSS grains is attained by hydrogen bonds among $\mathrm{HSO}_{3}$ groups of the PSS rich shell [2]. Impedance spectroscopy showed the electrical conductivity as percolation process between highly conductive PEDOT:PSS complexes in a matrix rich in PSS with three orders of magnitude lower conductivity [3].

PEDOT:PSS has extensively been used as buffer layer in organic optoelectronics and organic solar cells device applications [4,5]. Consequently, the degradation of PEDOT:PSS especially under atmospheric air containing oxygen and moisture, which influence the structure of this polymer, is crucial for the performance of organic electronic devices and it is still under investigation $[5,6]$. In this work the influence of oxygen and moisture of atmospheric air is studied by the comparison of the change of d.c. conductivity of two similar samples, the one under 
inert $\mathrm{He}$ and the other under atmospheric air.

\section{Experimental}

PEDOT:PSS films $120 \mathrm{~nm}$ thick, spin coated on pristine polyethylene terephthalene (PET) substrates were used. PEDOT:PSS was an aqueous dispersion (CLAVIOS PH 500, H.C. Starck, GmbH, Germany) with the ratio PEDOT/PSS to be $1: 2.5$ by weight.

A four probe method was used for the measurement of d.c. conductivity [7]. The temperature was monitored by an Oxford intelligent temperature controller (ITC4). A Keithley 2400 sourcemeter and a Keithley 182 sensitive digital voltmeter were used to control the current and voltage respectively and parameters were measured automatically via a PC.

\section{Results and Discussion}

In Figure 1 the $\sigma=\mathrm{f}(\mathrm{T})$ curves are shown for two similar PEDOT:PSS samples heated at a rate of $5 \mathrm{~K} / \mathrm{min}$, the one under inert He, the other in ambient atmosphere from 80 to $440 \mathrm{~K}$. Their shape reveals a competition between mechanisms increasing the conductivity and others which decrease it [5].

There are several factors which increase the conductivity with temperature: PEDOT:PSS is a semiconductor with energy gap $\mathrm{E}_{\mathrm{g}} \approx 1.8 \mathrm{eV}$ [8], so the carrier concentration is expected to increase with temperature. Besides, the heating of PEDOT films to $200^{\circ} \mathrm{C}(473 \mathrm{~K})$ revealed an improvement of the crystalline order, which makes easier the transport of carriers inside the grains. The simultaneous loss of water makes the hydrophilic PSS shell to shrink, which facilitates the tunneling of carriers between the grains [1].

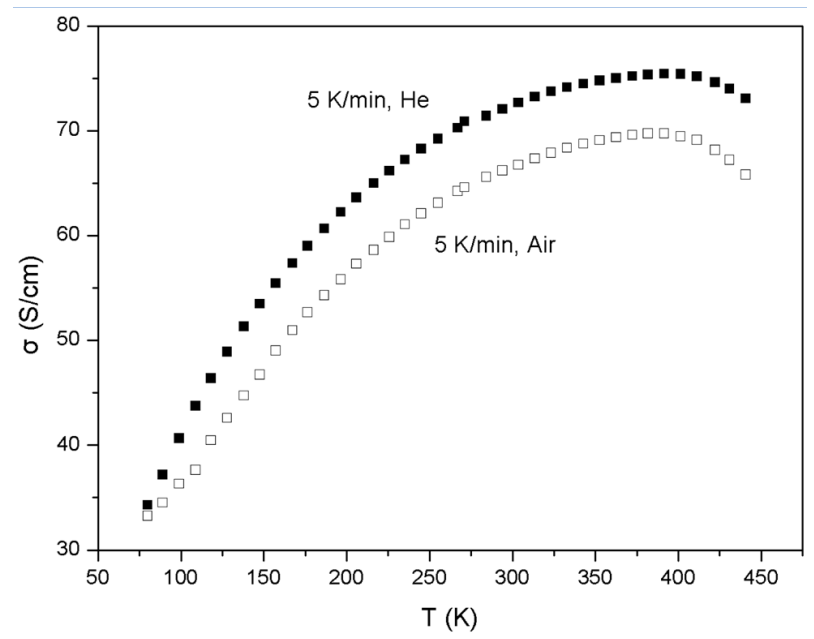

Figure 1. The change of the d.c. conductivity $\sigma$ vs $\mathbf{T}$ for two similar PEDOT:PSS films under inert helium and atmospheric air. The heating rate was $5 \mathrm{~K} / \mathrm{min}$.
Moreover, as the film is heated for longer periods, apart from the decrease of the intergrain distance mentioned before, an increment in the number of interparticle connections is observed, leading to an increase of the conductivity [9].

As temperature increases, the $\mathrm{Cl}^{-}$attached weakly to the hole polarons on the PEDOT oligomers, makes them to wander further away from the polaron vicinity increasing their mobility. At higher temperatures the rapture of the ionic bonds between PEDOT and PSS has been proposed, a process which improves order and produces highly conductive grains [4], whose size increases with thermal treatment, as atomic force microscopy has revealed [10].

On the other hand there are factors decreasing the conductivity as temperature increases. The insulating hydroscopic PSS surrounding the conductive grains can swell or shrink considerably depending on humidity [1].

Besides, hydrogen bonds between $\mathrm{HSO}_{3}$ groups of the PSS shells are broken as temperature increases making more difficult the transport of carriers between the PEDOT:PSS grains [2].

Heating PEDOT at $150^{\circ} \mathrm{C}(423 \mathrm{~K})$ under ambient atmosphere irreversible changes in the polymer main chain take place. Partial oxidation of the sulfur of the polymer backbone and the decomposition of the counter ion $\left(\mathrm{FeCl}^{-}\right)$resulting in decrease of conductivity [11].

It is obvious that the difference of $\sigma$ of the two similar samples at each $T$ the two curves of Figure 1, heated with the same rate but under different environments can give us an estimation of the role of oxygen and moisture contained in the atmospheric air on the structure of PEDOT:PSS. From Figure 1 and 2 it is shown that from 80 to $145 \mathrm{~K}$, for the heating rate of $5 \mathrm{~K} / \mathrm{min}$, although the conductivities increase for both environments with $\mathrm{T}$, the difference $\Delta \sigma$ raises abruptly, proving an intense degra-

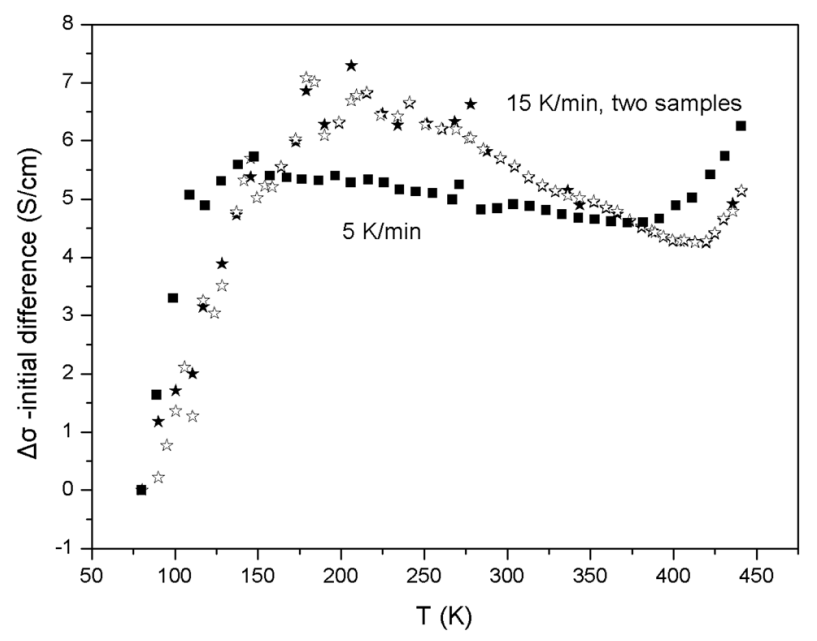

Figure 2 . The conductivity difference $\left(\sigma_{\mathrm{He}}-\sigma_{\text {air }}\right)$ vs $\mathrm{T}$ for two different heating rates: 5 and $15 \mathrm{~K} / \mathrm{min}$. 
dation of PEDOT:PSS from the oxygen and the moisture of the atmospheric air. The polymer is relatively resistant toward oxygen, though it proves sensitive to water because PSS is hydroscopic [12,13]. The abrupt increase of $\Delta \sigma$, i.e. the faster decrease of $\sigma$ under ambient conditions relatively to inert $\mathrm{He}$, may be attributed to the absorption of water, leading to the swelling of the insulating PSS shell surrounding the conducting PEDOT-rich grains [1]. For the faster heating rate of $15 \mathrm{~K} / \mathrm{min}$, the degradation advances slower, as it is shown in Figure 2 and it is extended from 80 to $200 \mathrm{~K}$ approximately. This is expected as the degradation process takes a certain time to be established. The intense scattering of the experimental points around $200 \mathrm{~K}$ for the $15 \mathrm{~K} / \mathrm{min}$ heating rate may be attributed to the fact that the absorption of water does not proceed with a smooth step under this quick heating rate.

From 145 to $395 \mathrm{~K}$ for $5 \mathrm{~K} / \mathrm{min}$ and from 205 to $410 \mathrm{~K}$ approximately for $15 \mathrm{~K} / \mathrm{min}$, the damage from the ambient atmosphere not only ceases to proceed, but exhibits a slight improvement, as it is indicated from the negative slope of the central "plateau" in Figure 2. This may be attributed to a situation in which there is an equal rate of absorption and loss of moisture in this temperature range.

The loss of moisture increases as temperature elevates, so the shrinking of the PSS insulating barriers gets more effective at higher temperatures decreasing slightly with $\Delta \sigma$.

For the higher heating rate, the loss of moisture is slower, so the difference $\Delta \sigma$ between the sample under He and the other under ambient atmosphere gets higher as the conductivity of the latter does not improve so much.

Broadband dielectric measurements on PEDOT:PSS films after thermal treatment under ambient temperature at $423 \mathrm{~K}\left(150^{\circ} \mathrm{C}\right)$ revealed that the PSS shell increases compared to pristine samples. So, the height of the potential barriers between the grains increases, reducing the conductivity [14]. Moreover, It has been found that for annealing temperatures between 373 and $473 \mathrm{~K}$ oxygen participates in the degradation of the PEDOT:PSS, as samples annealed under oxygen had a lower conductivity compared with samples annealed under nitrogen [15]. From Figure 1 it can be seen that the conductivity increases monotonically until a certain temperature $T_{m}$ and then decreases. This implies that the conductivity of PEDOT:PSS is a result of the competition between mechanisms which increase it and others which decrease it [5]. The characteristic temperature $\mathrm{T}_{\mathrm{m}}$ where the two competitive factors equilibrate takes the values $(384 \pm 4) \mathrm{K}$ and $(405 \pm 2) \mathrm{K}$ for the heating rates $5 \mathrm{~K} / \mathrm{min}$ and 15 $\mathrm{K} / \mathrm{min}$ respectively, independent from the surrounding atmosphere He or air. For $\mathrm{T}>\mathrm{T}_{\mathrm{m}}$ the degradation of the polymer prevails.

From Figure 2 we see that from about the same tem- peratures, $\Delta \sigma$ increases again, indicating that ambient environment decreases $\sigma$ more effectively than inert He. It is reasonable to assume that at this elevated temperature the role of oxygen is dominant.

TGA measurements and FTIR spectroscopy of PEDOD:PSS films revealed that there is no significant chemical changes up to the temperature of $523 \mathrm{~K}$ and there is an almost steady content of water for a wide range of temperatures [1]. Different oxidative reactions of the thiophene ring have been proposed [11] and for temperatures above $533 \mathrm{~K}$ PSS degrades reducing the PEDOT:PSS bulk conductivity.

To further investigate the differentiation of conductivity degradation near temperature $\mathrm{T}_{\mathrm{m}}$ where the two competitive factors equilibrate, the conductivity at $443 \mathrm{~K}$ $\left(170^{\circ} \mathrm{C}\right)$ versus the heating time $\mathrm{t}$ was measured on two films, one under inert He gas and another one under atmospheric air. The two isotherms $\sigma=\sigma(\mathrm{t})$ are shown in Figure 3.

Under inert He $\sigma$ increases monotonically with the heating time $t$, though there is a small "hump" after about $10 \mathrm{~min}$ of heating. The factors which increase conductivity prevail for all the heating time (60 min). The "hump" may be attributed to some intrinsic process, e.g. an abrupt improvement of crystallinity, which is counteracted later by heating induced disorder.

Under atmospheric oxygen and moisture (Figure 3) the isotherm has a completely different shape. There is an increase of $\sigma$ up to about $10 \mathrm{~min}$ (which corresponds to the intrinsic process taking place under $\mathrm{He}$ ) and then the destructive role of oxygen and moisture prevails [5].

Owing to its structure, PEDOT:PSS has been proved resistant toward molecular oxygen, but reacts relatively efficiently with water due to the hydrophilic character of PSS [12,13].

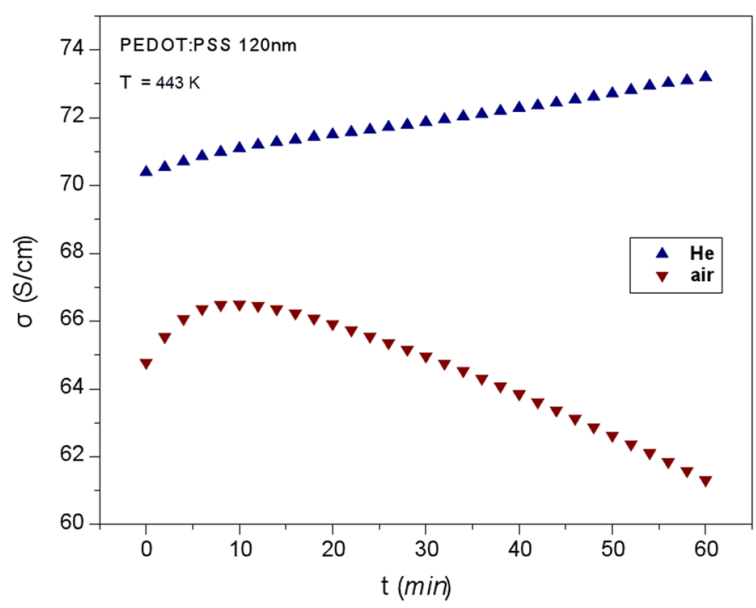

Figure 3. The change of the conductivity for the first heating of two PEDOT:PSS films, the one under inert helium, the other under atmospheric air at $443 \mathrm{~K}\left(170^{\circ} \mathrm{C}\right)$ for one hour. 
The decrease of $\sigma$ may be due to the break of hydrogen bonds between the conductive grains and the beginning of irreversible structural changes of the polymer chains.

In Figure 4 the isotherms at $443 \mathrm{~K}$ of the second heating are completely different from those of the first heating (Figure 3). The conductivity $\sigma$ under inert $\mathrm{He}$ remains practically constant with heating time, indicating that the first heating-cooling process stabilizes the morphology of the samples, so that the second heating does not affect it appreciably.

Under atmospheric air the isotherm (Figure 4) is almost linear, completely different from the corresponding curve in Figure 3. The stable morphology revealed under inert He deteriorates smoothly in the presence of oxygen and moisture.

\section{Conclusions}

The difference of the conductivity $\Delta \sigma$ at each temperature $\mathrm{T}$ between two similar samples of PEDOT:PSS heated with two rates 5 and $15 \mathrm{~K} / \mathrm{min}$ from 80 to $440 \mathrm{~K}$, the one under inert He and the other under ambient atmosphere, gives a measure of the role of oxygen and moisture on the conductivity.

The resulting curves exhibit three different regions: at low temperatures from 80 to $145 \mathrm{~K}$ for $5 \mathrm{~K} / \mathrm{min}$ and from 80 to $200 \mathrm{~K}$ for $15 \mathrm{~K} / \mathrm{min}$, an intense degradation of the sample under ambient atmosphere is revealed by the abrupt increase of the difference of the conductivities between He and atmospheric air. This may attribute to the absorption of moisture by hydroscopic PSS, a process which results in the swelling of the insulating of this polymer surrounding the conductive PEDOT-rich grains. PEDOT:PSS is more vulnerable to moisture than to oxygen because of the hydroscopic PSS.

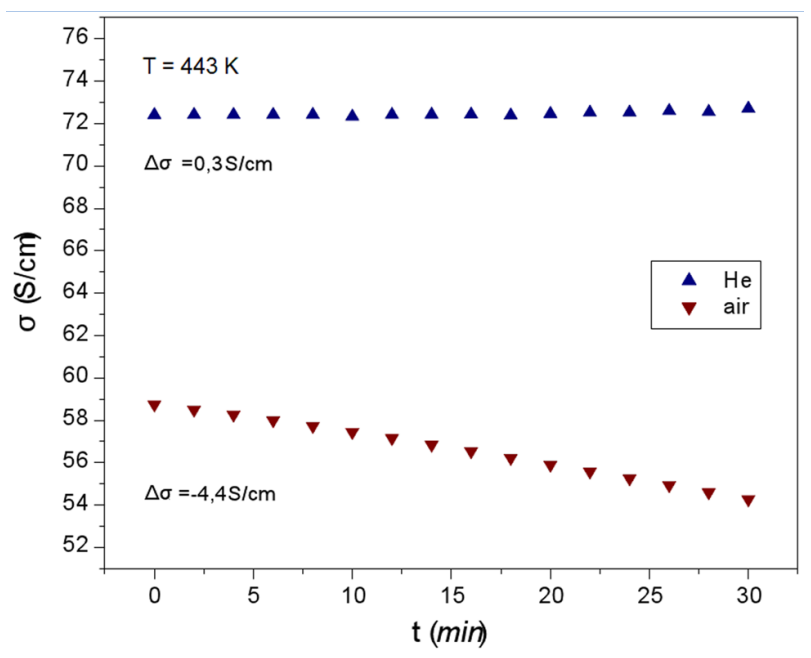

Figure 4. The conductivity change for the second heating of the two PEDOT:PSS films under inert helium and atmospheric air at $443 \mathrm{~K}\left(170^{\circ} \mathrm{C}\right)$ for thirty minutes.
In the second region of the $\Delta \sigma=\mathrm{f}(\mathrm{T})$ the conductivity difference $\Delta \sigma$ remains basically constant indicating a stabilization of the damage produced by oxygen and moisture. This may be explained by the equal rate of absorption and loss of water in this temperature range. Finally, for temperatures higher than 380 to $400 \mathrm{~K}$ the degradation increases again. It seems reasonable to assume that at these elevated temperatures degradation is due mainly to oxygen.

\section{REFERENCES}

[1] B. Friedel, P. E. Keivanidis, T. J. K. Brenner, A. Abrusci, C. R. McNeill, R. H. Friend and N. C. Greenham, "Effects of Layer Thickness and Annealing of PEDOT:PSS Layers in Organic Photodetectors,” Macromolecules, Vol. 42, No. 17, 2009, pp. 6741-6747. http://dx.doi.org/10.1021/ma901182u

[2] U. Lang, E. Müller, N. Naujoks and J. Dual, "Microscopical Investigations of PEDOT:PSS Thin Films," Advanced Functional Materials, Vol. 19, No. 8, 2009, pp. 12151220. http://dx.doi.org/10.1002/adfm.200801258

[3] T. Stöcker, A. Köhler and R. Moos, "Why Does the Electrical Conductivity in PEDOT:PSS Decrease with PSS Content? A Study Combining Thermoelectric Measurements with Impedance Spectroscopy,” Journal of Polymer Science Part B: Polymer Physics, Vol. 50, No. 14, 2012, pp. 976-983. http://dx.doi.org/10.1002/polb.23089

[4] E. Vitoratos, S. Sakkopoulos, E. Dalas, N. Paliatsas, D. Karageorgopoulos, F. Petraki, S. Kennou and S. A. Choulis, “Thermal Degradation Mechanisms of PEDOT:PSS," Organic Electronics, Vol. 10, No. 1, 2009, pp. 61-66. http://dx.doi.org/10.1016/j.orgel.2008.10.008

[5] E. Vitoratos, S. Sakkopoulos. N. Paliatsas, K. Emmanouil and S. A. Choulis, "Conductivity Degradation Study of PEDOT:PSS Films under Heat Treatment in Helium and Atmospheric Air,” Open Journal of Organic Polymer Materials, Vol. 2, No. 1, 2012, pp. 7-11. http://dx.doi.org/10.4236/ojopm.2012.21002

[6] C. L. Chochos and S. A. Choulis, "How the Structural Deviations on the Backbone of Conjugated Polymers Influence their Optoelectronic Properties and Photovoltaic Performance,” Progress in Polymer Science, Vol. 36, No. 10, 2011, pp. 1326-1414.

http://dx.doi.org/10.1016/j.progpolymsci.2011.04.003

[7] H. H. Wieder, "Laboratory Notes of Electrical and Galvanomagnetic Measurements,” Elsevier, Amsterdam, 1979.

[8] Y.-J. Lin, Y.-M. Chin, J.-C. Lin and Y.-C. Su, "Band Bending at the Conducting Polymer/Indium Tin Oxide Interfaces with and without Ultraviolet Treatment," Applied Surface Science, Vol. 256, No. 21, 2010, pp. 62596261. http://dx.doi.org/10.1016/j.apsusc.2010.03.151

[9] L. S. C. Pingree, B. A. MacLeod and D. S. Ginger, "The Changing Face of PEDOT:PSS Films: Substrate, Bias, and Processing Effects on Vertical Charge Transport," The Journal of Physical Chemistry C, Vol. 112, No. 21, 2008, pp. 7922-7927.

http://dx.doi.org/10.1021/jp711838h 
[10] Z. Xiong and C. Liu, "Optimization of Inkjet Printed PEDOT:PSS Thin Films through Annealing Processes,” Organic Electronics, Vol. 13, No. 9, 2012, pp. 15321540. http://dx.doi.org/10.1016/j.orgel.2012.05.005

[11] I. Winter, C. Reese, J. Hormes, G. Heywang and F. Jonas, "The Thermal Ageing of Poly(3,4-ethylenedioxythiophene). An Investigation by X-Ray Absorption and X-Ray Photoelectron Spectroscopy," Chemical Physics, Vol. 194, No. 1, 1995, pp. 207-213. http://dx.doi.org/10.1016/0301-0104(95)00026-K

[12] A. Elschner, "The Spectral Sensitivity of PEDOT:PSS Films," Solar Energy Materials \& Solar Cells, Vol. 95, No. 5, 2011, pp. 1333-1338. http://dx.doi.org/10.1016/j.solmat.2010.12.029

[13] K. Norman, M. V. Madsen, S. A. Gevorgyan and F. C. Krebs, "Degradation Patterns in Water and Oxygen of an Inverted Polymer Solar Cell," Journal of the American
Chemical Society, Vol. 132, No. 47, 2010, pp. 16883 16892. http://dx.doi.org/10.1021/ja106299g

[14] A. N. Papathanassiou, I. Sakellis, J. Grammatikakis, E. Vitoratos and S. Sakkopoulos, "Exploring Electrical Conductivity within Mesoscopic Phases of Semiconducting Poly(3,4-ethylenedioxythiophene):poly(4-styrene sulfonate) Films by Broadband Dielectric Spectroscopy,” Applied Physics Letters, Vol. 103, No. 12, 2013, pp. 123304 1-5. http://dx.doi.org/10.1063/1.4821101

[15] J. Huang, P. F. Miller, J. S. Wilson, A. J. de Mello, J. C. de Mello and D. D. C. Bradley, "Investigation of the Effects of Doping and Post-Deposition Treatments on the Conductivity, Morphology, and Work Function of Poly (3,4-ethylenedioxyphenethiophene)/Poly(styrene sulfonate) Films," Advanced Functional Materials, Vol. 15, No. 2, 2005, pp. 290-296.

http://dx.doi.org/10.1002/adfm.200400073 